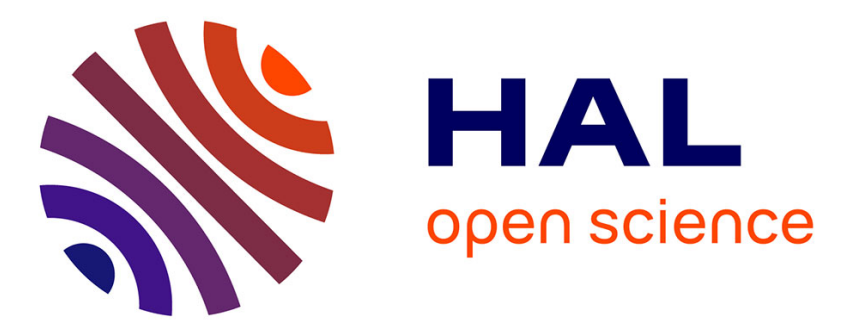

\title{
Motorcycle Riding Simulator Controllability and Simulator Sickness: A Proof-of-Concept System
}

Pauline Michel, Stéphane Espie, Samir Bouaziz

\section{To cite this version:}

Pauline Michel, Stéphane Espie, Samir Bouaziz. Motorcycle Riding Simulator Controllability and Simulator Sickness: A Proof-of-Concept System. Proceedings of the 11th International Conference on Simulation and Modeling Methodologies, Technologies and Applications (SIMULTECH 2021), Jul 2021, Online Streaming, France. pp.406-413, 10.5220/0010576704060413 . hal-03282210

\section{HAL Id: hal-03282210 https://hal.science/hal-03282210}

Submitted on 8 Jul 2021

HAL is a multi-disciplinary open access archive for the deposit and dissemination of scientific research documents, whether they are published or not. The documents may come from teaching and research institutions in France or abroad, or from public or private research centers.
L'archive ouverte pluridisciplinaire HAL, est destinée au dépôt et à la diffusion de documents scientifiques de niveau recherche, publiés ou non, émanant des établissements d'enseignement et de recherche français ou étrangers, des laboratoires publics ou privés. 


\title{
Motorcycle riding simulator controllability and Simulator Sickness: a proof-of-concept system
}

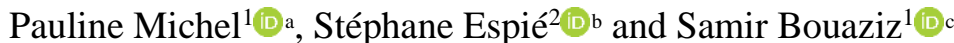 \\ ${ }^{1}$ Université Paris-Saclay, ENS Paris-Saclay, CNRS, SATIE, 91190 Gif-sur-Yvette, France \\ ${ }^{2}$ TS2-SATIE-MOSS, Univ Gustave Eiffel, IFSTTAR, F-77454 Marne-la-Vallée, France \\ \{pauline.michell,samir.bouaziz\}@universite-paris-saclay.fr, stephane.espie@univ-eiffel.fr
}

\begin{abstract}
Keywords: Driving Simulator, Simulator Sickness, Sensory fidelity, Hardware/software control, Human-simulator interaction, Sensor-actuator synchronization

Abstract: Driving a motorcycle relies on the feedback provided by several human sensory systems, on the one hand, and anticipation of the consequences of control actions, on the other hand. Driving simulators aim to create the illusion of driving by stimulating the driver's sensory systems. However, a significant number of drivers experience simulator sickness, which hinders the usefulness of driving simulators in their applications, such as driving behavior research or training / re-training. Simulator sickness occurrence is often attributed to sensory conflict. In this work, we propose an approach to understanding simulator sickness by considering the need for coherence between the complexity of the vehicle model and the complexity of the simulator from a hardware point-of-view, which constrains the fidelity of the reproduced sensory stimuli. We then describe the design of a proof-of-concept system that considers the particular issue of haptic feedback for the handlebars of a motorcycle-riding simulator. We will use this system in further experiments to demonstrate the impact of the coherence or mismatch of those two aspects on controllability and simulator sickness occurrence.
\end{abstract}

\section{INTRODUCTION}

Driving a vehicle requires the use of several human sensory systems: the visual, vestibular, haptic, and auditory are the main ones. Each of them plays a different role in the accomplishment of the driving task. In combination, they make it possible to estimate distance and speed, crucial parameters for driving, particularly for vehicle trajectory control, e.g. braking or collision avoidance. The coherence of the various sensory feedback, and the removal of any ambiguity between them, are ensured thanks to multisensory integration, i.e. fusion of this information carried out by the brain. The resulting information is the movement of the body in relation to the vehicle and its environment. In a driving task, this allows the driver to decide on a single interpretation of the current state of the vehicle being driven (position, speed, acceleration) (Kemeny et al., 2020), as well as the current state of other objects in the environment (e.g., other vehicles, pedestrians, road markings and signs, etc.).

The driver's sensory feedback is not sufficient to accomplish a driving task. The vehicle must also be guided to the desired destination (short-term and medium-term), which is an active closed-loop control-command and guidance task. The driving activity is traditionally divided into three task levels: strategical, tactical, and operational (Michon, 1985; see also Motte et al., 2019). To control the vehicle efficiently, i.e. to converge towards precise control, with minimal oscillations, a model of the controlled vehicle is required. In the case of human motor control, it is called the internal model and is learned and reinforced by experience (Wolpert et al., 2011; McNamee \& Wolpert, 2019; Pierella et al., 2019).

The goal of a driving simulator is to create the illusion of driving by stimulating the driver's sensory

a(D) https://orcid.org/0000-0002-9743-2402

b (D) https://orcid.org/0000-0002-3449-8279

c(D) https://orcid.org/0000-0002-6768-1723 
systems (Siegler et al., 2001; Fischer et al., 2016; Salisbury \& Limebeer, 2017). Inevitably, driving simulators provide only a subset of the sensory stimuli available in a real driving situation. The implementation of a high-fidelity dynamic vehicle model is a design constraint considered necessary to best reproduce real-world driving situations. This constraint, called physical validity (Malaterre \& Fréchaux, 2001; see also Faure, 2017, and Lobjois et al., 2021), is often taken into account without concern for the hardware architecture of the simulator and the sensory cues' fidelity. However, the poor or delayed restitution of a stimulus, similarly to the absence of its restitution, can lead to multisensory integration failing. Furthermore, all drivers do not use each sensory feedback in the same way. This makes it difficult to build a simulator suitable for all drivers and all purposes. Multisensory integration relies on the redundancy of sensory signals for disambiguation, but this is not always possible in driving simulators. This causes discomfort for drivers because it results in sensory conflict, which is identified and widely accepted as a cause of Simulator Sickness (SS) as well as Motion Sickness (MS) (Reason \& Brand, 1975).

In this work, we propose an approach to understanding SS, an adverse physiological reaction to a simulated driving situation. While MS primarily affects passengers in vehicles, all users of driving simulators can suffer from SS (Diels, 2016; Iskander et al., 2019). This is an essential concern for all driving simulator applications. We argue that SS comes from inadequacy between the complexity of the vehicle model and qualities of the cues provided to the driver $(*)$. Driving simulators often implement a high complexity vehicle model, e.g. with a large number of degrees of freedom and/or non-linearities, etc. Using a high complexity vehicle model, a driving simulator can simulate the dynamics of the real vehicle with high fidelity. However, if the architecture (HW/SW) of the simulator is not adapted accordingly, all of the sensory cues corresponding to the modeled physics cannot be provided to the driver. For example, in the case of motorcycle riding, the gyroscopic effect can be modeled but cannot be rendered using current technology. This inadequacy may lead to uncontrollability or poor controllability of the simulated vehicle that induces SS.

We focus on the complex case of reproducing haptic cues on the handlebars of a motorcycle driving simulator. We then describe the design of our ProofOf-Concept (POC) system, which we plan to use to test our hypothesis $(*)$ in various experiments. Our
POC system is a motorcycle driving simulator with haptic feedback on the handlebars. Its design takes into account the specific constraints of human sensory systems.

\section{ARCHITECTURE / MODEL MISMATCH AND SIMULATOR SICKNESS: OUR HYPOTHESIS}

Symptoms of SS may vary in type and intensity depending on the individual (Schweig et al., 2018). They can be separated into three clusters of symptoms: (1) oculomotor symptoms, (2) disorientation, and (3) nausea (Kennedy et al., 1993). Symptoms and intensity may also vary across situations for the same individual. In particular, they depend on the simulated task to accomplish (Kolasinski, 1995): for example, cornering is one of the most SS-inducing tasks, especially for the smallest curvature radii. Rich environments, such as urban junctions, are also particularly problematic. However, these situations cannot be excluded from driving simulators experiments. They are of great interest both in road safety research and public education initiatives and in training/retraining applications.

Experiencing symptoms of SS can affect the driver's task performance and/or reduce experiment duration (Money, 1970; Stoner et al., 2011; Liebherr et al., 2020). Besides, when a susceptible driver does not quit the experiment of their own accord, experiments are often only stopped when the experimenter is forced to (e.g., after the driver vomits). This constitutes a bad experience for the driver and can lead to them having a negative preconception of driving simulators in general. If the person agrees to participate in further driving simulator experiments, this tainted image can produce anxiety and pre-discomfort (Liebherr et al., 2021), which have been demonstrated to be negatively linked to SS (Bertin et al., 2004; Stelling et al., 2021). This negative preconception can also lead to definitive refusal of further participation in such experiments. Furthermore, the elderly population is particularly susceptible to SS. All of this induces involuntary "at the door" filtering of the population studied on driving simulators, i.e. the selection of the population that is not susceptible to SS. Recent studies report drop-out rates due to simulator sickness ranging from $5 \%$ to $30 \%$ when the participants are part of the general population (Balk et al., 2013; 
Liebherr et al., 2020; Saredakis et al., 2020). Additionnally, Matas et al. (2015) reported a drop-out rate of $59 \%$ for an experiment focusing on older adults. The results acquired thanks to driving simulators are hence often biased.

In a simulated driving simulation, as opposed to a real-world driving situation, the driver teleoperates a vehicle model, as represented in Figure 1. This is fundamentally different from driving a vehicle. Teleoperation control-command rules could be used.

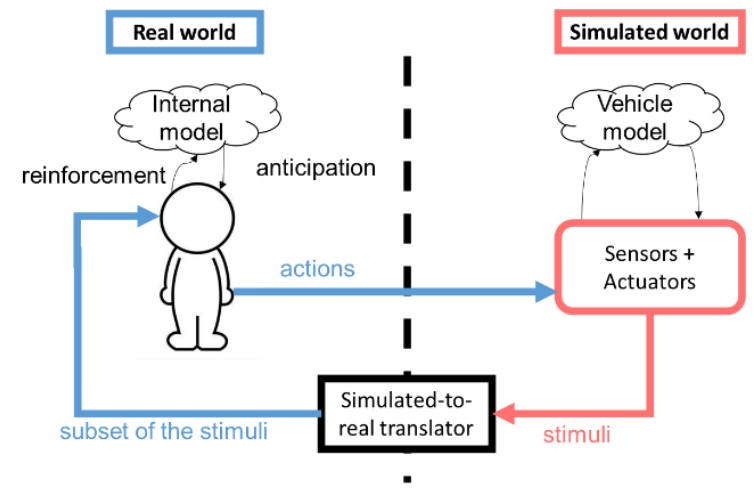

Figure 1: Illustration of the interactions between driver and driving simulator.

Motorcycle riding is more complex than driving a car. The rider controls the trajectory of their motorcycle through two torques: the roll torque and the steering torque, i.e. the torque applied by the rider on the motorcycle handlebars. The handlebars of a motorcycle serve a double action-perception purpose: the rider controls the system by interacting with them and they provide sensory feedback. Haptic cues on the handlebars are essential to the rider and significantly affect their riding behavior. For example, they provide feedback on the interaction between the motorcycle's tires and the road. However, measuring the rider's steer torque is a complex issue: when the rider exerts a torque on the motorcycle's handlebars, the steering column rotates. Moreover, at high speed, the variations of the angular position are of small amplitude. This means that any torque measure will not only reflect the torque applied by the rider but also the motorcycle's inherent dynamics. In motorcycle riding simulators, restitution of the sensory cues corresponding to haptic perception on the handlebars is therefore particularly complex and crucial. Poor or delayed restitution of haptic cues hinders the controllability of the virtual vehicle.
Motorcycles are inherently dynamically unstable: a rider needs to stabilize their motorcycle to ride it. That is why controllability is a crucial concern for motorcycle riding simulators, and similarly for car driving simulators. However, the research on the link between the controllability of a simulator and SS is still limited. Car-driving and motorcycle-riding simulators are currently used only in situations where they are fully controllable. However, as we previously mentioned, this means that driving simulation usage is deprived of situations that are of great interest such as driving at urban junctions.

A simulator being non-controllable may result in erratic, oscillating movements that produce uncontrolled image rotations, which have been shown to cause SS occurrence (Golding, 2006; Cohen et al., 2019). Moreover, experiencing control difficulties may prompt the driver to feel anxious and uncomfortable, feelings which, as mentioned above, are also negatively linked to SS.

Our opinion is that a mismatch between the complexity of the vehicle model and the fidelity of the sensory stimuli that correspond to it prevents the driver from being able to adequately control the virtual vehicle, which then induces SS. As discussed, SS occurrence and SS symptoms severity are intrinsically linked to psychological validity, which we believe should be the goal in designing driving simulators for road safety research or training applications. In the following, we focus on the design of a POC system for this hypothesis, with a special interest in providing haptic feedback.

\section{OUR PROOF-OF-CONCEPT SYSTEM}

Because driving a motorcycle involves several human sensory systems, each of which the precise role in the driving task depends on the rider, deciding what sensory stimuli is reproduced and how is a complex issue. However, human sensory systems have inherent time and frequency sensitivities, as well as physical and biochemical limitations that need to be taken into account. For example, sensory receptor and neuromuscular dynamics, nerve conduction, and neural processing altogether are responsible for a time delay between the instant when a sensory stimulus (or stimuli) is applied and the instant when the control response begins. Time delays respectively introduced 
by the visual and haptic system are presented in Table 1. In this section, we describe the design of our POC system under these constraints.

Table 1: Sensory delays characteristic of the visual and haptic systems (Nash et al., 2016).

\begin{tabular}{|c|c|}
\hline Sensory system & Sensory delay \\
\hline Visual & $100 \mathrm{~ms}-560 \mathrm{~ms}$ \\
\hline Haptic & $\begin{array}{c}>34 \mathrm{~ms} \\
\text { or }>48 \mathrm{~ms} \\
\text { (depending on } \\
\text { the receptors) }\end{array}$ \\
\hline
\end{tabular}

\subsection{System architecture}

Our POC system, represented in Figure 2 provides the driver with haptic cues using motorcycle handlebars mechanically coupled to a CanisDrive-20A-160-AM$\mathrm{H}$-SIE servo actuator, pictured in Figure 3. Our system also provides visual cues using a Virtual Reality (VR) headset, the HTC Vive Pro system. The goal for the driver is to control the trajectory of a virtual motorcycle through a virtual scene using this bimodal feedback.

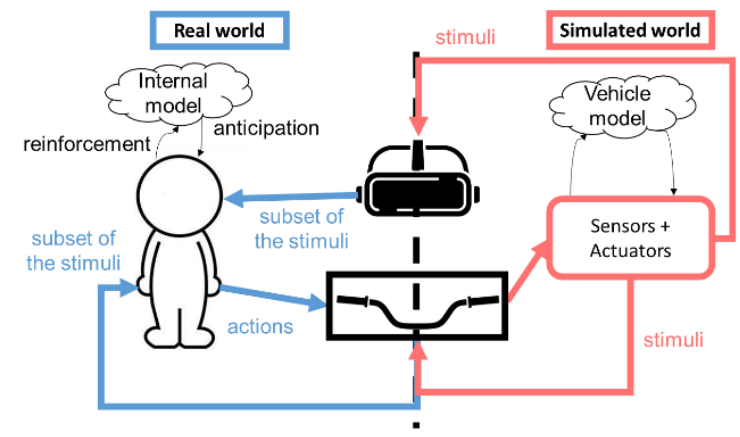

Figure 2: Schematic representation of the interactions between the driver and our POC system.

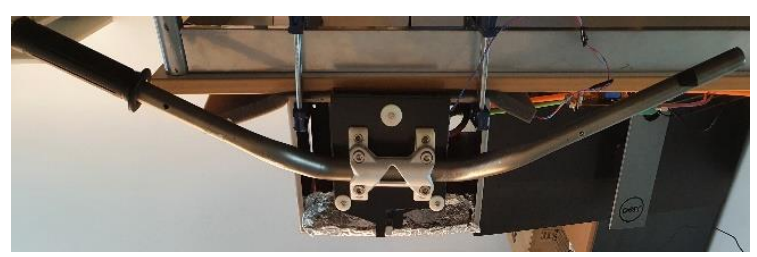

Figure 3: Haptic feedback motorcycle handlebars used in our POC system.

We use a distributed architecture, as represented by its deployment diagram in Figure 4. It is implemented across:

- $\quad$ a PC embedding an Intel ${ }^{\circledR}$ Core $^{\mathrm{TM}}$ i7-8700 CPU @ 3.20GHz and a Nvidia GeForce GTX 1060 responsible for the computing of the dynamic model of the simulated system (e.g., bicycle or motorcycle). The dynamic model is computed at a frequency of $1 \mathrm{kHz}$. This PC is also responsible for generating the images for visual rendering;

- a lab-made board embedding a mbed (LPC1768) microcontroller and a FieldProgrammable Gate Array (FPGA), a DE0Nano board, responsible for the data acquisition of the torque applied to the handlebars by the driver;

- a lab-made board embedding a STM32F446 microcontroller and a DE0-Nano board, responsible for generating haptic cues in conjunction with a YukonDrive-1021-ADO servo controller.

This distributed architecture guarantees the stability of the calculation, sampling, and transmission frequencies imposed by the constraints we will discuss in this part. Frequency jitters would negatively impact the controllability of the riding simulator, which we want to avoid per our hypothesis (*).

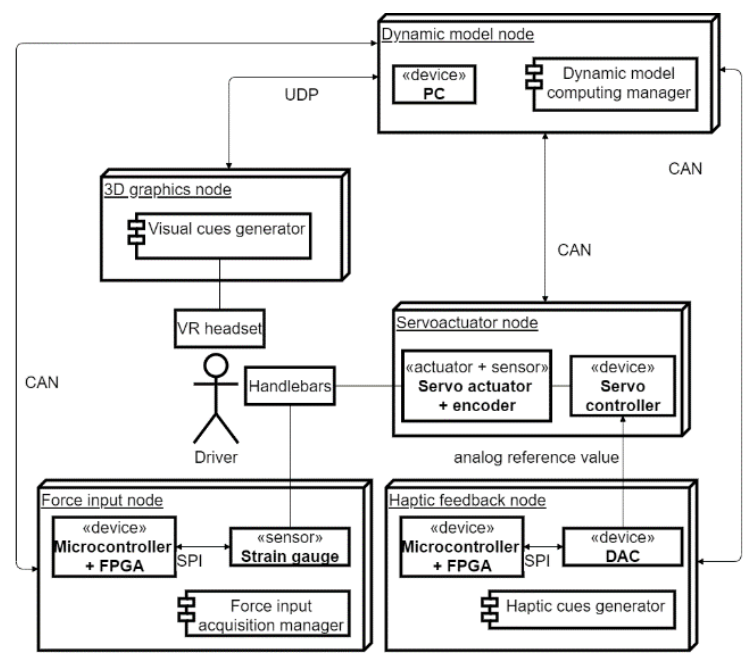

Figure 4: Deployment diagram of our POC system.

The servo actuator and servo controller are isolated with special care to avoid electromagnetic interference between them and the VR headset's display.

\subsection{Human control input}

In motorcycle riding simulation, as previously stated, the rider does not drive a real motorcycle, but remotely operates a motorcycle model by interacting with a physical system. In the case of our POC system, the driver controls the trajectory of the 
motorcycle model by acting on the handlebars. The resulting torque on the steering column is an input of the motorcycle model and needs to be measured. The servo actuator we use has been specifically picked because of its high gear ratio $(\mathrm{R}=160)$, which makes it non-manually reversible, ensuring the separation of the system's inherent dynamics and the human action on the system. The torque exerted by the driver on the handlebars can thus be measured directly using a strain gauge.

The torque data acquisition board was designed so that the embedded FPGA can sample the measure of up to four sensors via SPI communication. In our application, only one channel is used. The sampled measures are sent to the embedded microcontroller after being requested, also via SPI communication. Both SPI blocks implemented on the FPGA use a $10 \mathrm{MHz}$ SCLK frequency for data transfer. The microcontroller filters the strain gauge data using an Infinite Impulse Response (IIR) filter, specifically a $2^{\text {nd }}$ order low-pass Butterworth numerical filter. The filter data is then re-transmitted via $\mathrm{CAN}$ bus, with a CAN bus speed of $1 \mathrm{Mbits} / \mathrm{s}$.

\subsection{Visual cueing}

Visual cues projected into the Head-Mounted Display (HMD) are computed in real-time by a 3D graphics generator, the Unity3D engine with the OpenXR plugin. Relevant model outputs (e.g., position, speed, acceleration) are sent over Ethernet using UDP at a frequency of $90 \mathrm{~Hz}$. Using an HMD will also allow us to implement audio cueing in further work.

For visual rendering, the first time-related constraint that was taken into account is the image refresh rate. A constant, sufficient refresh rate is necessary for the driver to operate under the impression of continuous, fluid visual motion. 30 frames per second (FPS) is commonly defined as the acceptable minimum frame rate for this purpose. However, the images projected in the case of driving simulators often include vehicles moving at high speeds, which require a higher refresh rate for the animation to appear continuous and fluid. Moreover, a variable refresh rate induces image flickering, as well as instability of the virtual environment when using an HMD. These visual effects result in erratic, oscillating movements of the simulated motorcycle from the point-of-view of the driver. In our system, visual cues are generated by the $3 \mathrm{D}$ graphics generator at a constant refresh rate of 90FPS.
As presented in Table 1, the visual system is characterized by a sensory delay between the perception of a visual change in the environment and control response ranging from $100 \mathrm{~ms}$ to $560 \mathrm{~ms}$ (Nash et al., 2016). However, drivers are sensible to much lower transport delay, i.e. time difference between the instant of a control-command action - in the case of our system, turning the handlebars - and system response. For vision, system response is a change in the visual scene. When the transport delay introduced by a system is greater than an acceptable transport delay, the system becomes more difficult to control or even uncontrollable. It also causes uneasiness for the driver. In our application, feedback is bimodal: visual-tactile. This impacts acceptable latencies for both the visual and haptic feedback. The maximum acceptable transport delays are system-, task- and person-dependent (Attig et al., 2017). For a simulated driving task, which is a time-critical task, the maximum acceptable visual latency reported in the literature is 50ms (Frank et al., 1988; Padmos \& Milders, 1992). As previously stated, in our system, the VR environment simulation runs at 90FPS. Assuming that all computations are performed within one frame, this frame rate alone introduces a latency of $11 \mathrm{~ms}$. However, there are additional software and hardware sources of transport delay. For the HTC Vive Pro used in conjunction with the Unity3D game engine, Le Chénéchal and Chatel-Goldman (2018) found a mean transport delay of $31.33 \mathrm{~ms}$. This is an acceptable visual latency for a simulated driving task. However, transport delay does not only depend on the visual rendering sub-system, but also on the human haptic cueing sub-system, which will be discussed in the next subsection.

In conclusion of this section, the design of the visual cueing sub-system of our POC system takes into account physiological constraints specific to vision in the particular context of having to accomplish a simulated driving task. Consideration of these constraints allows us to avoid involuntary noncontrollability of the simulated motorcycle and image flickering and/or oscillations. This will enable us to test our hypothesis $\left(^{*}\right)$ by changing visual feedback modalities without the risk of uncontrolled changes in those modalities caused by the system.

\subsection{Haptic cueing}

Haptic perception is divided into two dependent sensory sub-modalities (Reed \& Ziat, 2018):

- $\quad$ kinesthesia, i.e. the perception of the body's movement thanks to proprioceptive sensors 
that provide feedback on efforts endured by the muscles and on the angular position of the body's limbs;

- tactile perception, or sense of touch, i.e., the perception of the skin's interaction with the environment (pressure, vibration, temperature, texture, roughness, etc.) thanks to cutaneous surface feedback on the material properties of objects in contact with the body and the angular position of its limbs.

The mechanoreceptors, specific sensory receptors located in the different layers of the skin and the joints and muscles, are respectively responsible for tactile perception and kinesthesia. There are several types of mechanoreceptors, which are each sensitive to specific stimuli of different frequencies. Riding a motorcycle using handlebars activates three particular types of mechanoreceptors, the characteristics of which are summarized in Table 2. Their respective frequency sensitivities impose design constraints for the frequency-related parameters of haptic cueing in our POC system. The torque exerted by the driver on the handlebars is sampled at a frequency of $500 \mathrm{~Hz}$. This is coherent with the frequency sensitivities of the mechanoreceptors involved in the driving task. It also complies with teleoperation control-command rules that specify a haptic refresh rate in the range of $500 \mathrm{~Hz}$ to $1 \mathrm{kHz}$ to ensure the stability and transparency of the haptic interaction. This loop frequency guarantees the controllability of the system and thus of the simulated motorcycle.

The haptic rendering board was specifically designed for this application so that the embedded FPGA can sample the encoder data directly from its serial transmission by the servo controller. Like the strain gauge data acquisition board, the FPGA and microcontroller of this board communicate using SPI with a $10 \mathrm{MHz}$ SCLK frequency. Encoder data, i.e. position and speed of the handlebars, is re-transmitted over CAN bus at a $1 \mathrm{~ms}$ period by the microcontroller. This information is used as inputs of the dynamic model. This sampling and transmission frequency is compliant with the haptic loop constraints previously established.
Table 2: Characteristics (stimulation type sensitivity and frequency sensitivity) of the mechanoreceptors relevant to the driving task (Hale \& Stanney, 2004).

\begin{tabular}{|c|c|c|}
\hline Mechanoreceptors & $\begin{array}{c}\text { Stimulation type } \\
\text { (relevant for the } \\
\text { driving task) } \\
\text { sensitivity }\end{array}$ & $\begin{array}{c}\text { Frequency } \\
\text { sensitivity }\end{array}$ \\
\hline Pacinian corpuscles & $\begin{array}{c}\text { Vibration, } \\
\text { acceleration }\end{array}$ & $\begin{array}{c}100 \mathrm{~Hz}- \\
1 \mathrm{kHz}\end{array}$ \\
\hline Ruffini endings & $\begin{array}{c}\text { lateral force, } \\
\text { motion }\end{array}$ & $0.4 \mathrm{~Hz}-$ \\
& $\begin{array}{c}100 \mathrm{~Hz} \\
\text { direction, static } \\
\text { force }\end{array}$ \\
\hline Meissner corpuscles & $\begin{array}{c}\text { Velocity, grip } \\
\text { control }\end{array}$ & $2 \mathrm{~Hz}-$ \\
& & $40 \mathrm{~Hz}$ \\
\hline
\end{tabular}

We have described what feedback is transmitted to the PC that computes the dynamic model, and how and when it is. Our haptic feedback actuator is speed controlled using a Proportional Integral (PI) controller. However, in our implementation, the speed output of the model is not applied directly as speed reference but is first corrected using the current position error. This position-speed dual control avoids position derivation due to incremental speed tracking error and numerical integration. The block diagram representation of the position-speed control is given in Figure 5. For clarity, model inputs are not exhaustively represented, but they have been described in the text.

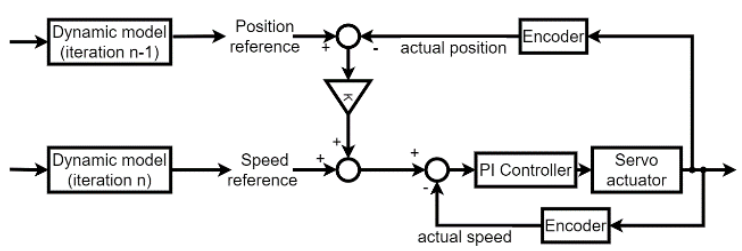

Figure 5: Block diagram representation of the servo actuator's position-speed control.

Similarly to vision, haptic perception allows for a maximum acceptable transport delay between the driver trying to turn the handlebars and the handlebars effectively turning. Even though nerve messages have a longer distance to travel between the arms and hands and the brain than between the eyes and the brain, delays characteristic of haptic perception are significantly shorter than delays characteristic of vision (as evidenced in Table 1; see also Cameron et al., 2014; Crevecoeur et al., 2016). The maximum acceptable haptic delay varies significantly on the task, the system, and the person (Kaber \& Zhang, 2011). Our objective was the 
minimization of haptic latency in our system. CAN bus communication introduces a well-known delay that corresponds to the duration of a CAN frame. The maximum duration of a CAN base frame is around $134 \mu \mathrm{s}$ (for an 8 bytes data frame). The CAN arbitration process also adds another delay that is difficult to quantify. This is why the speed reference value for the servo actuator is transmitted via an analog input directly to the servo controller. Speed is thus controlled while minimizing additional delay in the system control that affects both haptic and visual latency.

To summarize, we designed the haptic rendering sub-system of our POC system by taking into account physiological constraints specific to haptic perception, such as haptic sensory receptors frequency sensitivities and minimal haptic latency. This ensures that this sub-system induces no involuntary non-controllability of the simulated motorcycle. This will allow us to test our hypothesis (*) by changing haptic feedback modalities without risk of uncontrolled changes in those modalities caused by reasons inherent to the system. Furthermore, our POC system provides the rider with good quality haptic feedback on motorcycle handlebars, which we believe is necessary to ensure the controllability of any motorcycle driving simulator.

\section{CONCLUSIONS}

We argue that Simulator Sickness comes from inadequacy between the complexity of the vehicle model and the fidelity of the sensory cues to be reproduced. We have taken a special interest in motorcycle riding simulators and in particular in the issue of providing good quality haptic feedback on the motorcycle handlebars. Indeed, this feedback significantly affects the simulator's controllability and is not often taken into account.

We aim to demonstrate the cruciality of the coherence between both of those aspects. To do so, we have designed a Proof-Of-Concept system that takes into account the specific constraints of human sensory systems. This design philosophy, detailed in this work, will thus allow us to modulate visual and/or haptic feedback. By doing so, we will be able to compare the results in terms of (1) controllability and task performance and (2) anxiety, discomfort, and eventual SS symptoms severity of a motor control task when the complexity of the vehicle model and the fidelity of the sensory cues (a) when they are coherent and (b) when they are mismatched. The exploration of our hypothesis in the case of a "simple" task using this POC system will be our next step. Our haptic feedback subsystem will allow us to explore the impact of the adequacy of the motorcycle dynamic model's complexity with the complexity of the simulator architecture on trajectory control, presence, and SS occurrence in a future experiment. We plan to compare these aspects for coherent and mismatched modalities defined by: (1) two dynamic motorcycle models of different complexity, and (2) disabled or enabled haptic restitution for the same motorcycle riding simulator platform.

\section{REFERENCES}

Attig, C., Rauh, N., Franke, T., \& Krems, J. F. (2017). System latency guidelines then and now - is zero latency really considered necessary? In D. Harris (Ed.), Engineering Psychology and Cognitive Ergonomics 2017, Part II, LNAI 10276 (2-14).

Bertin, R. J. V., Guillot, A., Collet, C., Vienne, F., Espié, S., \& Graf, W. (2004). Objective measurement of simulator sickness and the role of visual-vestibular conflict situations: a study with vestibular-loss (areflexive) subjects (poster). Driving Simulation Conference.

Cameron, B. D., De la Malla, C., \& López-Moliner, J. (2014). The role of differential delays in integrating transient visual and proprioceptive information. Frontiers in Psychology, 5(50).

Cohen, B., Dai, M., Yakushin, S. B., \& Cho, C. (2019). The neural basis of motion sickness. Journal of Neurophysiology, 121(3), 973-982.

Crevecoeur, F., Munoz, D. P., \& Scott, S. H. (2016). Dynamic multisensory integration: somatosensory speed trumps visual accuracy during feedback control. Journal of Neuroscience, 36(33), 8598-8611.

Diels, C., \& Bos, J. E. (2016). Self-driving carsickness. Applied Ergonomics, 53, 374-382.

Faure, V. (2017). Les simulateurs de conduite : évaluation de la validité psychologique sous l'angle de la charge mentale [Doctoral dissertation]. Université ParisSaclay.

Fischer, M., Seefried, A., \& Seehof, C. (2016). Objective motion cueing test for driving simulators. Proceedings of Driving Simulation Conference, 41-50.

Frank, L. H., Casali, J. G., \& Wierwille, W. W. (1988). Effects of visual display and motion system delays on operator performance and uneasiness in a driving simulator. Human Factors, 30(2), 201-217.

Golding, J. F. (2006) Motion sickness susceptibility. Autonomic Neuroscience: Basic and Clinical, 129(1-2), 67-76

Hale, K., \& Stanney, K. (2004). Deriving haptic design guidelines from human physiological, psychophysical, 
and neurological foundations. Proceedings of IEEE Conference on Computer Graphics and Applications, 24(2), 33-39.

Iskander, J., Attia, M., Saleh, K., Nahavandi, D., AboBakr, A., Mohamed, S. M. K., Asadi, H., Khosravi, A., Lim, C., \& Hossny, M. (2019). From car sickness to autonomous car sickness: a review. Transportation Research Part F-Traffic Psychology and Behavior, 62, 716-726.

Kaber, D. B., \& Zhang, T. (2011). Human factors in virtual reality system design for mobility and haptic task performance. Reviews of Human Factors and Ergonomics, 7(1), 323-366.

Kemeny, A., Chardonnet, J. R., \& Colombet, F. (2020). Self-motion perception and cybersickness. In Getting Rid of Cybersickness (31-62).

Kennedy, R. S., Lane, N. E., Berbaum, K. S., \& Lilienthal M. G. (1993). Simulator sickness questionnaire: an enhanced method for quantifying simulator sickness. The International Journal of Aviation Psychology, 3(3), 203-220.

Kolasinski, E. (1995). Simulator sickness in virtual environments (Technical Report $\mathrm{n}^{\circ} 1027$ ). US Army Research Institute for the Behavioral and Social Sciences.

Le Chénéchal, M., \& Chatel-Goldman, J. (2018). HTC Vive Pro time performance benchmark for scientific research. Proceedings of International Conference on Artificial Reality and Telexistence and Eurographics Symposium on Virtual Environments, 81-84.

Liebherr, M., Schweig, S., Brandtner, A., Averbeck, H., Maas, N., Schramm, D., \& Brand, M. (2020). When virtuality becomes real: relevance of mental abilities and age in simulator adaptation and dropouts. Ergonomics, 63(10), 1271-1280.

Liebherr, M., Mueller, S. M., Schweig, S., Maas, N., Schramm, D., \& Brand, M. (2021). Stress and simulated environments: insights from physiological marker. Frontiers in Virtual Reality, 2.

Lobjois, R., Faure, V., Désiré, L., \& Benguigui, N. (2021). Behavioral and workload measures in real and simulated driving: do they tell us the same thing about the validity of driving simulation? Safety Science, 134.

Malaterre, G., \& Fréchaux, J. (2001). Etude de la validité des simulateurs de conduite par comparaison de tâches réalisées en situation réelle et en simulation. In $\mathrm{V}$. Cavallo \& C. Berthelon (Eds.), Facteurs perceptifs dans les activités de transport (149-156).

Matas, N. A., Nettelbeck, T., \& Burns, R. (2015). Dropout during a driving simulator study: a survival analysis. Journal of Safety Research.

McNamee, D., \& Wolpert, D. M. (2019). Internal models in biological control. Annual Review of Control, Robotics, and Autonomous Systems, 2(1), 339-364.

Michon, J. A. (1985). A critical view of driver behavior models: what do we know, what should we do? In L. Evans \& R. C. Schwing (Eds.), Human behavior and traffic safety (485-520).

Money, K. E. (1970). Motion sickness. Physiological Review, 50(1), 1-39.
Motte, C., Lappi, O., Giles, O., Markkula, G., Mars, F., \& Wilkie, R. (2019). Getting back into the loop: the perceptual-motor determinants of successful transitions out of automated driving. Human Factors, 61(7), 1037 1065.

Nash, C. J., Cole, D. J., \& Bigler, R. (2016). A review of human sensory dynamics for application to models of driver steering and speed control. Biological Cybernetics, 110(2-3), 91-116.

Padmos, P., \& Milders, M. V. (1992). Quality criteria for simulator images: a literature review. Human Factors, 34(6), 727-748

Pierella, C., Casadio, M., Mussa-Ivaldi, F. A., \& Solla, S. A. (2019). The dynamics of motor learning through the formation of internal models. PLOS Computational Biology, 15(12).

Reason, J. T., \& Brand, J. J. (1975). Motion Sickness. London: Academic Press.

Reed, C. L., \& Ziat, M. (2018). Haptic perception: from the skin to the brain. In Reference Module in Neuroscience and Biobehavioral Psychology.

Salisbury, I., \& Limebeer, D. (2017). Motion cueing in high-performance vehicle simulators. Vehicle System Dynamics, 55(6), 775-801.

Saredakis, D., Szpak, A, Birckhead, B., Keage, H. A. D., Rizzo, A., \& Loetscher, T. (2020). Factors associated with virtual reality sickness in head-mounted displays: a systematic review and meta-analysis. Frontiers in Human Neuroscience, 14.

Schweig, S., Liebherr, M., Schramm, D., Brand, M. \& Maas, N. (2018). The impact of psychological and demographic parameters on simulator sickness. In Proceedings of $8^{\text {th }}$ International Conference on Simulation and Modeling Methodologies, Technologies and Applications - SIMULTECH, 91-97.

Siegler, I., Reymond, G., Kemeny, A., \& Berthoz, A. (2001). Sensorimotor integration in a driving simulator: contribution of motion cueing in elementary driving task. Proceedings of Driving Simulation Conference, 21-32.

Stelling, D., Hermes, M., Gerrit, H., Mittelstädt, J., Niedermeier, D., Schudlik, K., \& Duda, H. (2021). Individual differences in the temporal progression of motion sickness and anxiety: the role of passengers' trait anxiety and motion sickness history. Ergonomics.

Stoner, H., Fisher, D., \& Mollenhauer, M. Jr. (2011). Simulator and scenario factors influencing simulator sickness. Handbook of Driving Simulation for Engineering, Medicine and Psychology, 14-1 - 14-24.

Wolpert, D. M., Diedrischsen, J., \& Flanagan, J. R. (2011). Principles of sensorimotor learning. Nature Reviews Neuroscience, 12(12), 739-751. 